\title{
Technical note: A new day- and night-time Meteosat Second Generation Cirrus Detection Algorithm MeCiDA
}

\author{
W. Krebs, H. Mannstein, L. Bugliaro, and B. Mayer \\ Deutsches Zentrum für Luft- und Raumfahrt (DLR) \\ Received: 25 June 2007 - Published in Atmos. Chem. Phys. Discuss.: 26 July 2007 \\ Revised: 30 October 2007 - Accepted: 29 November 2007 - Published: 18 December 2007
}

\begin{abstract}
A new cirrus detection algorithm for the Spinning Enhanced Visible and Infra-Red Imager (SEVIRI) aboard the geostationary Meteosat Second Generation (MSG), MeCiDA, is presented. The algorithm uses the seven infrared channels of SEVIRI and thus provides a consistent scheme for cirrus detection at day and night. MeCiDA combines morphological and multi-spectral threshold tests and detects optically thick and thin ice clouds. The thresholds were determined by a comprehensive theoretical study using radiative transfer simulations for various atmospheric situations as well as by manually evaluating actual satellite observations. The cirrus detection has been optimized for midand high latitudes but it could be adapted to other regions as well. The retrieved cirrus masks have been validated by comparison with the Moderate Resolution Imaging Spectroradiometer (MODIS) Cirrus Reflection Flag. To study possible seasonal variations in the performance of the algorithm, one scene per month of the year 2004 was randomly selected and compared with the MODIS flag. $81 \%$ of the pixels were classified identically by both algorithms. In a comparison of monthly mean values for Europe and the North-Atlantic MeCiDA detected $29.3 \%$ cirrus coverage, while the MODIS SWIR cirrus coverage was $38.1 \%$. A lower detection efficiency is to be expected for MeCiDA, as the spatial resolution of MODIS is considerably better and as we used only the thermal infrared channels in contrast to the MODIS algorithm which uses infrared and visible radiances. The advantage of MeCiDA compared to retrievals for polar orbiting instruments or previous geostationary satellites is that it permits the derivation of quantitative data every $15 \mathrm{~min}, 24 \mathrm{~h} \mathrm{a}$ day. This high temporal resolution allows the study of diurnal variations and life cycle aspects. MeCiDA is fast enough for near real-time applications.
\end{abstract}

Correspondence to: B. Mayer

(bernhard.mayer@dlr.de)

\section{Introduction}

Clouds are one of the most important components of the global climate system. According to IPCC (2007), "cloud feedbacks remain the largest source of uncertainty" in the climate sensitivity of the Earth. Cirrus clouds, in particular, have attracted special attention because they can provide a positive net cloud radiative forcing (Hansen et al., 1997). Cirrus clouds or high ice clouds cover approximately $21 \%$ (Rossow and Schiffer, 1999) to 33\% (Wylie et al., 2005) of the Earth - this number of course depends on the definition of "cirrus cloud" and in particular on the detection limit of the observing instrument. Changes in cirrus cloud coverage may significantly alter the Earth's climate. The detection and quantification of cirrus clouds and the understanding of their formation and dissipation is therefore important for the understanding of the atmosphere and the prediction of climate change.

The impact of cirrus clouds on the radiation budget of the Earth depends strongly on their optical properties but also on solar zenith angle and surface albedo. Their radiative effect is a delicate balance between reflection of shortwave radiation and trapping of longwave radiation: While the first effect usually dominates for optically thick clouds, thin cirrus clouds may cause a net warming effect, depending on their microphysical properties and ice water content (Meerkötter et al., 1999). Cirrus coverage may have a diurnal cycle (Wylie and Woolf, 2002). Monitoring of cirrus clouds with geostationary satellite instruments allows the investigation of diurnal variations as well as the possibility of observation of life cycle aspects. However, thin cirrus represents a challenge to satellite retrieval methods because of its weak contrast with the underlying surface and/or atmosphere.

Satellite instruments are an ideal means to derive cloud properties on a global scale. Various studies have addressed the retrieval of cirrus clouds from satellite instruments and

Published by Copernicus Publications on behalf of the European Geosciences Union. 
for some of them, data are operationally available. In particular, the MODIS (MODerate Resolution Imaging Spectroradiometer) instrument on board the Terra and Aqua satellites with its 36 spectral channels is one of the most accurate passive instruments to derive cirrus cloud properties from radiance observations (Ackerman et al., 1998; Platnick et al., 2003; King et al., 2003). New active lidar instruments in space (e.g. the Geoscience Laser Altimeter System GLAS (Mahesh et al., 2004; Hart et al., 2005) or CALIPSO which is part of the A-Train) may provide even more accurate information, in particular about semi-transparent cirrus. All these instruments have one common disadvantage: They are employed on polar-orbiting platforms which constrains the availability of data to the local overpass time. Instruments like MODIS provide at least one observation per day while sophisticated instruments like GLAS and CALIPSO only provide a very limited coverage of the Earth. Geostationary instruments, on the other hand, provide information frequently (typically every hour or faster), but they typically have only a few broadband channels at low spatial resolution. Nevertheless, the big advantage of their high time resolution has been exploited in several studies, e.g. (Minnis and Smith Jr., 1998; Feijt et al., 2000).

The Spinning Enhanced Visible and Infra-Red Imager (SEVIRI) onboard the geostationary satellite Meteosat Second Generation (MSG) (Schmetz et al., 2002) combines the advantages of both worlds to some degree. MSG/SEVIRI, which became operational at the end of January 2004, provides data with a high temporal resolution of $15 \mathrm{~min}$. SEVIRI comprises twelve spectral bands, seven of which are pure thermal infrared and provide data at day and night with a spatial resolution of $3 \mathrm{~km} \times 3 \mathrm{~km}$ at the sub-satellite point. In particular, two channels (the water vapor channels WV6.2 and WV7.3 centered at $6.2 \mu \mathrm{m}$ and $7.3 \mu \mathrm{m}$ respectively) are sensitive to upper tropospheric water vapor, three are window channels (the infrared channels IR8.7, IR10.8, and IR12.0 at $8.7,10.8$ and $12.0 \mu \mathrm{m}$ ), one (IR9.7) includes the ozone absorption band at $9.7 \mu \mathrm{m}$ and one (IR13.4) is located in the $\mathrm{CO}_{2}$ absorption band at $13.4 \mu \mathrm{m}$. The three pure solar channels were not used for our analysis because they are obviously only available during day time. The mixed solar/thermal channel at $3.9 \mu \mathrm{m}$ was also excluded as it is "contaminated" by solar radiation during day time and therefore would also introduce a day/night difference into the derived product. Based on the seven thermal channels we developed an algorithm MeCiDA (Meteosat Cirrus Detection Algorithm) using morphological and multi-spectral threshold techniques. In contrast to more general MSG/SEVIRI cloud classification schemes (e.g. Derrien and Le Gleau, 2005) we specifically aim at the detection of cirrus clouds during day and night and optimized the algorithm for that purpose. MeCiDA combines well-known cirrus detection methods e.g. the semi-transparent thin cirrus or "split-window" test which uses the brightness temperature difference $T_{10.8}-T_{12.0}$ (Inoue, 1985; Kriebel et al., 2003) and new techniques. De- tailed radiative transfer simulations have been performed to fully exploit the unique spectral opportunities of SEVIRI. Using only the thermal channels the algorithm offers the possibility to observe cirrus clouds at day and night with the same detection scheme. Since it is fast enough for near realtime applications, MeCiDA allows the generation of homogeneous time series of cirrus cloud coverage maps which can improve the current understanding of the formation, evolution and dissipation of this important cloud type.

For validation of the algorithm, we compared MeCiDA results with the standard MODIS cirrus cloud product. MODIS is a key instrument aboard the NASA Terra and Aqua polar orbiting satellites. It acquires data in 36 spectral bands with a spatial resolution up to $250 \mathrm{~m} \times 250 \mathrm{~m}$, some of which had been specifically designed for the detection of cirrus clouds. MeCiDA cirrus masks have been compared with the Cirrus Reflectance Flag from the MOD06 L2 Products (Platnick et al., 2003; King et al., 2003; Ackerman et al., 1998) for the North-Atlantic, Europe, and North-Africa. The spatial resolution of this product is $1 \mathrm{~km} \times 1 \mathrm{~km}$ - much higher than the SEVIRI resolution, which is approximately $4 \mathrm{~km} \times 5 \mathrm{~km}$ in mid-latitudes.

The paper is organised as follows: Sect. 2 describes the basic theory and methods, in particular the definition of "cirrus cloud" with respect to what is actually detected by MeCiDA, the physics behind the detection of cirrus clouds, and the radiative transfer model which we used for the study. Section 3 presents the MeCiDA algorithm in detail. Section 4 shows the results of the validation. Finally, summary and conclusions are presented in Sect. 5.

\section{Theory and methods}

\subsection{Cirrus classification}

Cirrus (latin for "curl of hair") clouds cover a wide range of optical and microphysical properties. A definition of "cirrus" is therefore required to illustrate what the algorithm actually detects. The World Meteorological Organization (WMO) gives the following definitions (World Meteorological Organization, 1975, 1987):

Cirrus. Detached clouds in the form of white, delicate filaments or white or mostly white patches or narrow bands. These clouds have a fibrous (hair-like) appearance, or a silky sheen, or both.

Cirrocumulus. Thin, white patch, sheet or layer of cloud without shading, composed of very small elements in the form of rains, ripples etc., merged or separate, and more or less regularly arranged; most of the elements have an apparent width of less than one degree.

Cirrostratus. Transparent, whitish cloud veil of fibrous (hair-like) or smooth appearance, totally or partly covering the sky, and generally producing halo phenomena.

These definitions are entirely morphological and are based on the visual appearance at the ground during daytime. 
"Sub-visible cirrus" is well recognized in meteorology but is currently not included in the WMO classification (Lynch et al., 2002). In addition to natural cirrus, there are manmade clouds, in particular "aircraft contrails" and "contrail cirrus" into which a persistent contrail eventually evolves. Even if the morphology leads to an obvious classification, and the morphological properties are used by the developed algorithm to detect cirrus clouds, morphology is only the first step to physical classification. "Were this not the case, then whales would still be called fish and planets would be classified as stars ..." (Lynch et al., 2002). Temperature, altitude, phase, and optical properties are commonly used for the classification of clouds from satellite data (Rossow and Schiffer, 1999).

MeCiDA aims to detect clouds, the optical properties of which are dominated by ice, with high cloud top height, or equivalently, low cloud top temperature. As any passive instrument in non-limb geometry, MeCiDA fails to detect subvisible cirrus but rather detects those clouds with a significant impact on the radiation budget of the Earth, with an optical thickness in the order of magnitude 0.1 or more depending on the atmospheric and surface conditions as well as on the viewing geometry. In this paper these clouds are simply called "cirrus". This also includes deep convective clouds if they are cold enough. MeCiDA classifies them as thick ice clouds which is a reasonable approach as deep convective clouds are usually topped by a cirrus layer.

\subsection{Radiative transfer simulations}

The new multi-spectral threshold techniques used by our algorithm have been developed on the basis of detailed radiative transfer calculations. Equivalent brightness temperatures for the thermal SEVIRI channels have been simulated for different atmospheric and surface conditions with the radiative transfer package libRadtran (Mayer and Kylling, 2005). libRadtran offers a flexible interface to setup the atmospheric and surface conditions as well as a choice of different radiative transfer equation solvers. For the simulation of radiances or brightness temperatures in this paper we selected the DISORT 2.0 solver by Stamnes et al. (1988) with 16 streams which allows accurate simulations of radiances. Molecular absorption is accounted for by the LOWTRAN atmospheric band model (Pierluissi and Peng, 1985) adopted from the SBDART radiative transfer code (Ricchiazzi and Gautier, 1998) which uses a three-term exponential sum fit with a resolution of $20 \mathrm{~cm}^{-1}$. Each SEVIRI channel is simulated with 15 spectral grid points, weighted with the filter function, and integrated over wavelength. Ice cloud single scattering properties in the thermal IR were parameterised according to Fu et al. (1998) which includes the single scattering properties of hexagonal ice columns for a wide range of effective radii. Profiles of temperature, pressure, and trace gas concentrations were taken from Anderson and Hall (1989). libRadtran has been successfully validated in several model inter- comparison campaigns and by direct comparison with observations, e.g. (Van Weele et al., 2000; Mayer et al., 1997).

For this study, two sets of simulations were used: (1) a systematic variation of various input parameters, to study the dependence on specific variables, like the surface temperature, cloud top height, etc.; (2) a test data set with a random variability of all relevant input data to cover a wide range of possible conditions. For the latter, 10000 different combinations of atmospheric conditions were used as input:

- profiles of pressure, temperature, water vapor, ozone concentration and other trace gases were taken from the TIGR-3 (Thermodynamic Initial Guess Retrieval) data set (Chevallier et al., 1998);

- the ice cloud optical thickness was varied between 0 and 10, the ice particle effective radius between 10 and $45 \mu \mathrm{m}$, with a bottom height between 6 and $10 \mathrm{~km}$ and a geometrical thickness of $0.5-2 \mathrm{~km}$;

- in $50 \%$ of the cases, a water cloud was added below the cirrus, with optical thickness between 5 and 50, droplet radius $5-15 \mu \mathrm{m}$, cloud bottom height $1-2 \mathrm{~km}$, and cloud geometrical thickness $0.5-2 \mathrm{~km}$;

- the surface emissivity was assumed to be 1 in all cases and the surface skin temperature was calculated by adding a random $\pm 10 \mathrm{~K}$ to the temperature of the lowest level of the atmospheric profile; in a future study, the spectral variation of the emissivity should also be considered, but for the application in this paper the role of the surface is comparatively small because in all tests the "cloudless" background is subtracted from the data anyway.

For each atmopheric data set, brightness temperatures were calculated for satellite zenith angles between 0 and $78^{\circ}$, in equidistant steps of 0.02 in the cosine of the satellite zenith angle. Thus, preference is given to larger satellite viewing angles in which we were interested most (Europe and the North-Atlantic). A total of 410000 data points was obtained (41 viewing angles for 10000 atmospheric conditions), 50\% with and 50\% without water clouds below the cirrus cloud. This test data set covers a wide range of atmospheric and surface conditions and forms an ideal basis for optimizing and evaluating threshold tests.

\subsection{Cirrus detection basics}

Clouds in satellite images are usually characterized by their higher reflectance and lower brightness temperature than the underlying surface and/or atmosphere. However, cloud types such as thin, semi-transparent cirrus are difficult to detect because of insufficient contrast. Here we present some general detection principles for thin and thick ice clouds. We concentrate on the thermal channels because we aim at a consistent algorithm for day- and night-time and thereby abandon the 

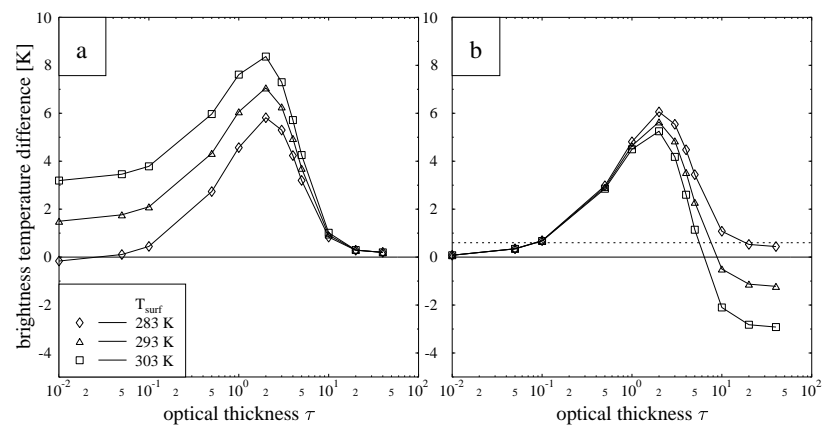

Fig. 1. Simulated brightness temperature difference of SEVIRI channels IR10.8 and IR12.0 for the mid-latitude summer atmosphere, a cirrus cloud between 10 and $11 \mathrm{~km}$, effective particle radius $6 \mu \mathrm{m}$, and satellite zenith angle $60^{\circ}$. (a) Temperature difference as a function of the cloud optical thickness for three different surface temperatures; (b) same, but after subtraction of the respective cloudless temperature difference (= corrected BTD).

additional information provided by the solar channels which is available only during day time and which would introduce systematic differences between the results for day and night.

An imager aboard a satellite provides radiances or equivalent brightness temperatures for specific spectral bands. The simple conceptual model by Kidder and Vonder Haar (1995) allows to separate the different contributions to the brightness temperature or radiance at top-of-atmosphere. For cloudless conditions, the spectral radiance $L$ is approximately

$L \approx \epsilon_{S} \cdot B\left(T_{S}\right) t_{A}+B\left(T_{A}\right)\left(1-t_{A}\right)$

and in the case with a cirrus cloud

$L \approx \epsilon_{S} \cdot B\left(T_{S}\right) t_{A} t_{C}+B\left(T_{A}\right)\left(1-t_{A}\right) t_{C}+B\left(T_{C}\right)\left(1-t_{C}\right)$

where $B(T)$ is the Planck function, $T_{S}$ the surface temperature, $\epsilon_{S}$ the surface emissivity, $T_{A}$ the effective temperature of the atmosphere, $T_{C}$ the temperature at cirrus cloud top, $t_{A}$ the atmospheric transmittance, and $t_{C}$ the transmittance of the cirrus cloud. The transmittances of course depends on wavelength and satellite viewing angle, and the Planck function $B(T)$ strongly depends on wavelength. For opaque cirrus $\left(t_{C} \approx 0\right)$ the right side of $(2)$ reduces to

$L \approx B\left(T_{C}\right)$

The simplest way to detect cold ice clouds is a single channel threshold technique where the observed brightness temperature $T_{\lambda}$ at a given wavelength $\lambda$ is compared to some threshold

$T_{\lambda}<T_{\lambda, \text { threshold }}$

This method works well for opaque clouds, but for optically thin or semi-transparent clouds, the radiance or brightness temperature includes contributions from the cirrus cloud as well as from the surface and atmosphere below. The thinner the cirrus cloud, the closer is the radiance to its cloudless value (Eqs. 1 and 2) and the harder is the cirrus cloud to detect. For the detection of thin cirrus, several multi-spectral techniques have been described in the literature, e.g. the semi-transparency or "split-window" test based on two channels at 10.8 and $12.0 \mu \mathrm{m}$ (Inoue, 1985):

$T_{10.8}-T_{12.0}>T_{\text {threshold }}$

The physical basis of this algorithm is the difference in the single scattering properties of ice clouds at these two wavelengths. Figure 1a shows as an example the "classical" split-window test for IR10.8 and IR12.0 channels. The three curves correspond to three different surface temperatures which clearly have an effect on the temperature difference. To reduce the effect of surface and atmosphere (first and second term in 2), one may subtract the corresponding difference for cloudless conditions:

$T_{10.8}-T_{12.0}-\left(T_{10.8, \text { cloudless }}-T_{12.0, \text { cloudless }}\right)>T_{\text {threshold }}$

Such differences will be called "corrected brightness temperature differences" or "corrected BTD" in the following. Figure 1b shows the result of the subtraction: all curves coincide now for small optical thickness and thin to moderatelythick ice clouds may be detected using a single threshold value. The dashed line indicates the threshold of $0.6 \mathrm{~K}$ which is actually used in the MeCiDA algorithm. With this threshold, clouds with an optical thickness between 0.1 and 5 should be detected, but the actual detection limit of course depends on the profiles of temperature and trace gas concentrations, the cloud top height, the particle size, and the satellite zenith angle. The threshold was chosen by evaluating a large set of radiative transfer calculations for a wide range of conditions. The required cloudless brightness temperature differences needs to be determined either from numerical weather prediction model or reanalysis data (e.g. Feijt et al., 2000) or by searching the warmest pixel in the neighbourhood of the given pixel and assuming it to be cirrus-free. The second method was used in several detection tests and is part of the MeCiDA Algorithm.

In addition to the spectral characteristics of cirrus clouds our algorithm exploits the morphological properties of cirrus in the water vapor channels like WV7.3 and WV6.2 on SEVIRI. Water vapor channels are ideally suited for cirrus detection, as absorption and emission by atmospheric water vapor effectively "shields" surface and lower clouds. In the water vapor channels the impact of the surface temperature can therefore be neglected as can be seen in Fig. 2, left panel. The radiance at top-of-atmosphere is emitted from water vapor and high clouds only. The horizontal distribution of the integrated water vapor path is usually rather smooth while cirrus clouds often exhibit considerable small-scale variability and thus cause a high frequency disturbance in the image, see left panel of Fig. 2: Here the cold structured cirrus cloud 


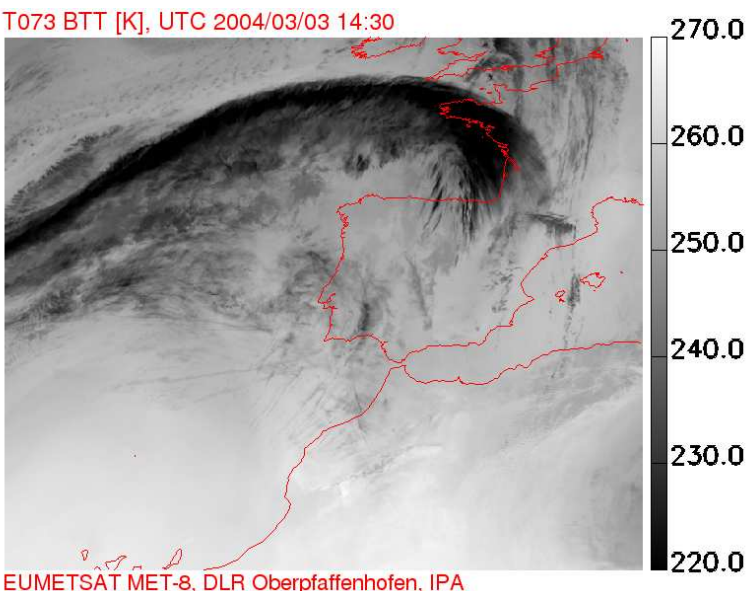

EUMETSAT MET-8, DLR Oberpfaffenhofen, IPA

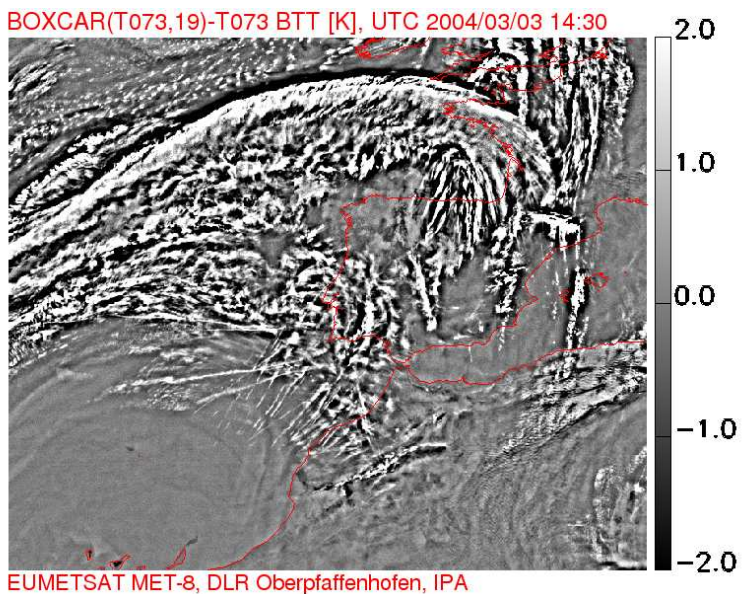

Fig. 2. (Left) brightness temperature image of SEVIRI water vapor channel WV7.3 over South Western Europe and the West coast of Africa on 3 March 2004 at 14:30 UTC; a cirrus band is clearly visible by its darker color (lower temperature); (right) high-pass filtered image by subtracting a $19 \times 19$ pixel average (boxcar filter or moving average) from the original image.

is clearly visible before the smooth water vapor background in the WV7.3 channel. Using a high-pass filter the cloud can be extracted from the image: By subtracting a smoothed image from the original image, the high frequency part of the image is extracted (right panel of Fig. 2) and can again be automatically detected by applying a threshold. In MeCiDA we use either a Gaussian filter or a boxcar filter which is simply a moving average over a certain area. This test is similar to the well known spatial coherence test (e.g. Saunders and Kriebel, 1988) usually applied to window channel brightness temperatures over ocean.

The combination of two types of algorithms (threshold and morphological) by a logical AND has the advantage that thin cirrus is detected using different, more or less independent, principles. This allows to tune the single test to a high detection efficiency without getting too many false alarms. Figure 3 shows the combination of the brightness temperature difference test, the morphological test, and the simple threshold which together form Test 1 (see below). For example the morphological test alone sometimes misclassifies structured low level clouds and surface features like coastlines as cirrus, but these are usually not detected by the brightness temperature difference test and are therefore excluded. The optically thick and homogeneous cirrus clouds which are not detected by the thin cirrus tests are finally added by a simple threshold test, see the detailed description of Test 1 in the next section.

\section{The MeCiDA Algorithm}

MeCiDA uses the seven infrared SEVIRI channels and combines morphological and multi-spectral threshold tests to detect thick and thin ice clouds. For a better understanding the algorithm is divided into six sub-groups or detection schemes (test 1-test 6). These sub-groups are described in detail in this section. Each of the six tests is a full cirrus test in its own and could be used alone. In order to increase the detection efficiency of our method, we combine the results of all tests with a logical OR, that is, if at least one of the six tests classifies a pixel as cloudy, it is considered cloudy. In consequence, each test needs to have a low "false alarm rate". For several tests, thresholds were determined on the basis of comprehensive radiative transfer calculations, in particular for the temperature-difference tests. For others, thresholds were defined empirically by visual inspection of satellite images, in particular the thresholds for the morphological tests. Test 1: split-window 10.8-12.0 $\mu \mathrm{m}$ and morphology $7.3 \mu \mathbf{m}$

Test 1 uses the split-window temperature difference $T_{10.8}-T_{12.0}$ to detect thin cirrus. As shown in Fig. 1a, the threshold temperature is sensitive to surface temperature and the atmospheric profiles. To reduce the influence of surface and atmosphere, the corresponding cloudless brightness temperature difference is estimated using the warmest neighbourhood pixel from a $3 \times 3,9 \times 9$, or $19 \times 19$ pixel area around the pixel under consideration and subtracted from the temperature difference. We used three different areas because, for example, close to coastlines a too large area might fail if a thin cirrus is detected over ocean while the corresponding cloudless pixel is over land and in consequence the cloudless temperature difference doesn't match the conditions under the cirrus. On the other hand, if the area is too small, the probability is larger that it actually doesn't contain a cloudless pixel. This is true not only for coastlines but for all kinds of heterogeneous areas.

Figure 4 shows the application of this test to our test data set described in Sect. 2.2. It is immediately obvious from the top plot that the test is highly sensitive to cirrus with optical thickness between about 0.1 and 2 . Optically thinner cirrus 

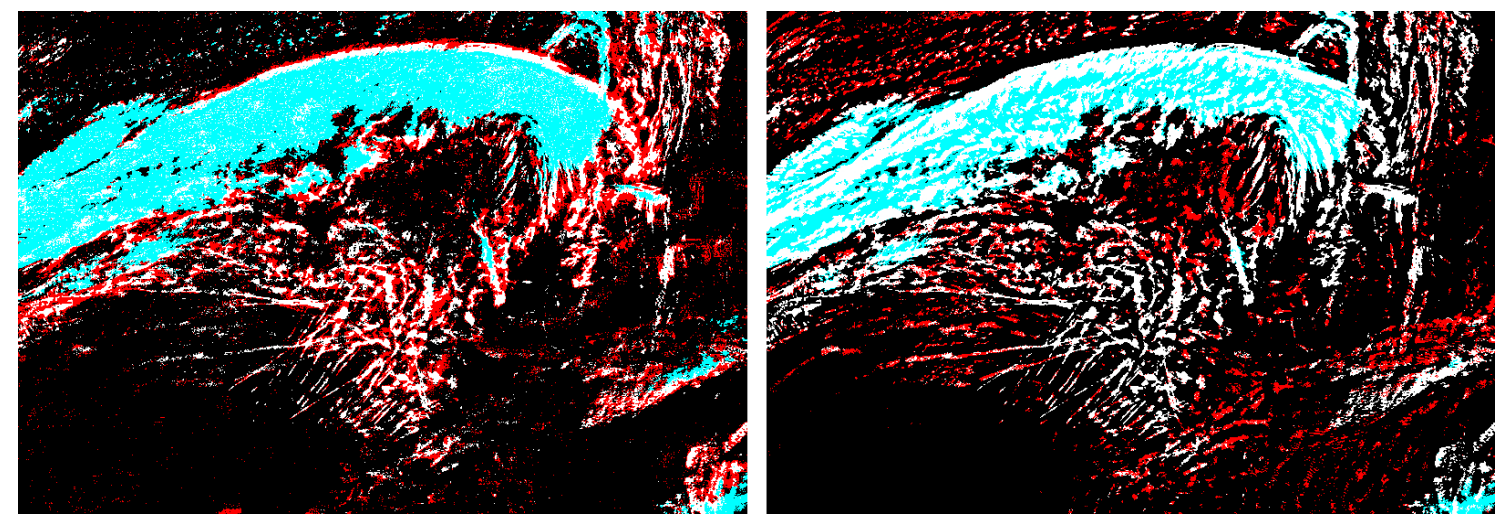

Fig. 3. (Left) white and red pixels are those detected by the corrected brightness temperature test $T_{10.8}-T_{12.0}$; red pixels were detected by the corrected brightness temperature test $T_{10.8}-T_{12.0}$, but not by the morphological test on WV73 - they are excluded by the logical AND; the cyan pixels were added by the threshold test on $T_{6.2}-T_{7.3}$ (Test $1 \mathrm{~b}$ ) to the corrected brightness temperature test; white and cyan together are all pixels detected by Test 1 . (Right) white and red pixels are those detected by the morphological test on WV73; red pixels were detected by the morphological test on WV73 but not by the corrected brightness temperature test $T_{10.8}-T_{12.0}-$ they are excluded by the logical AND; the cyan pixels were added by the threshold test on $T_{6.2}-T_{7.3}$ (Test $1 \mathrm{~b}$ ) to the morphological test; white and cyan together are all pixels detected by Test 1 .

is not detected because the impact on the brightness temperature differences is too small and optically thick ice clouds are also missed because the brightness temperature difference vanishes and the corrected brightness temperature difference approaches $T_{10.8 \text {,cloudless }}-T_{12.0 \text {,cloudless. Please note that for }}$ the determination of the corrected BTD for the cases with a water cloud below the cirrus, we decided to subtract the corresponding temperature difference for the atmosphere including the water cloud, rather than the completely cloudless atmosphere. This corresponds for example to the situation of a cirrus above an extended stratus deck. The middle plot shows a histogram of corrected brightness temperature differences for two optical thicknesses 0.1 and 1 . While a considerable fraction of the optically thin clouds is missed by the test, $90 \%$ of the optically thicker clouds are captured by the test. Finally, the lower plot shows the detection efficiency of the test as a function of optical thickness. The test works best for optical thickness between 0.5 and 2 , with decreasing detection efficiency for thinner and thicker clouds. The detection efficiency is hardly affected by low water clouds which are included in the "all-sky" data shown in grey but not in the "without water cloud" data in blue. Interestingly, the detection efficiency is even slightly higher in the all-sky case. This is explained by the fact that the optical properties of water clouds are very similar in the IR10.8 and IR12.0 channels for which reason the brightness temperature difference for a water cloud is smaller than the corresponding brightness temperature difference for a cloudless atmosphere. As water clouds shield the surface and the fraction of the atmosphere below the cloud, they effectively reduce the variability of the background brightness temperature difference which slightly increases the detection efficiency. We need to add a few words on the methodology used: The simulated test data set gives only a first idea of the performance of the threshold tests of the retrieval. The test data set is based on a variety of atmospheric and surface conditions which probably covers most of the natural variability. However, optimum conditions are assumed for the retrieval: First, we assume a perfect instrument, neglecting possible biases and noise in the radiance observation. Second, we also assume that the correct background temperature is retrieved while in reality it is assumed that the maximum brightness temperature in a given area around the pixel under consideration is actually the corresponding cloudless value for the pixel under consideration. The algorithm thus implicitly requires that a cloudless pixel is actually available in the area, and that the cloudless value is constant within the area. For these reasons, the model data set is considered a useful tool for the development of the detection algorithm but cannot replace a careful validation of the product with real data which we will show later in this paper. Obviously, the test data set also cannot be used to validate the morphological tests.

The application of the described split-window test to real data indicates some sensitivity to partially cloudy pixels for example at the edges of low level water clouds. To prevent this misclassification, the test result is combined with structural information from the water vapor channel WV7.3 which is not sensitive to low level water clouds: The WV7.3 image is high-pass filtered by subtracting the $19 \times 19$ pixel mean value (boxcar filtered image) from the original image (see Fig. 2). Combining these two tests with a logical AND, we detect all pixels with thin-to-moderately thick structured cirrus clouds while horizontally homogeneous wide-spread clouds with larger optical thickness might be missed. To include those, a third test is added with a logical OR using the difference between the two water vapor channels WV7.3 and 

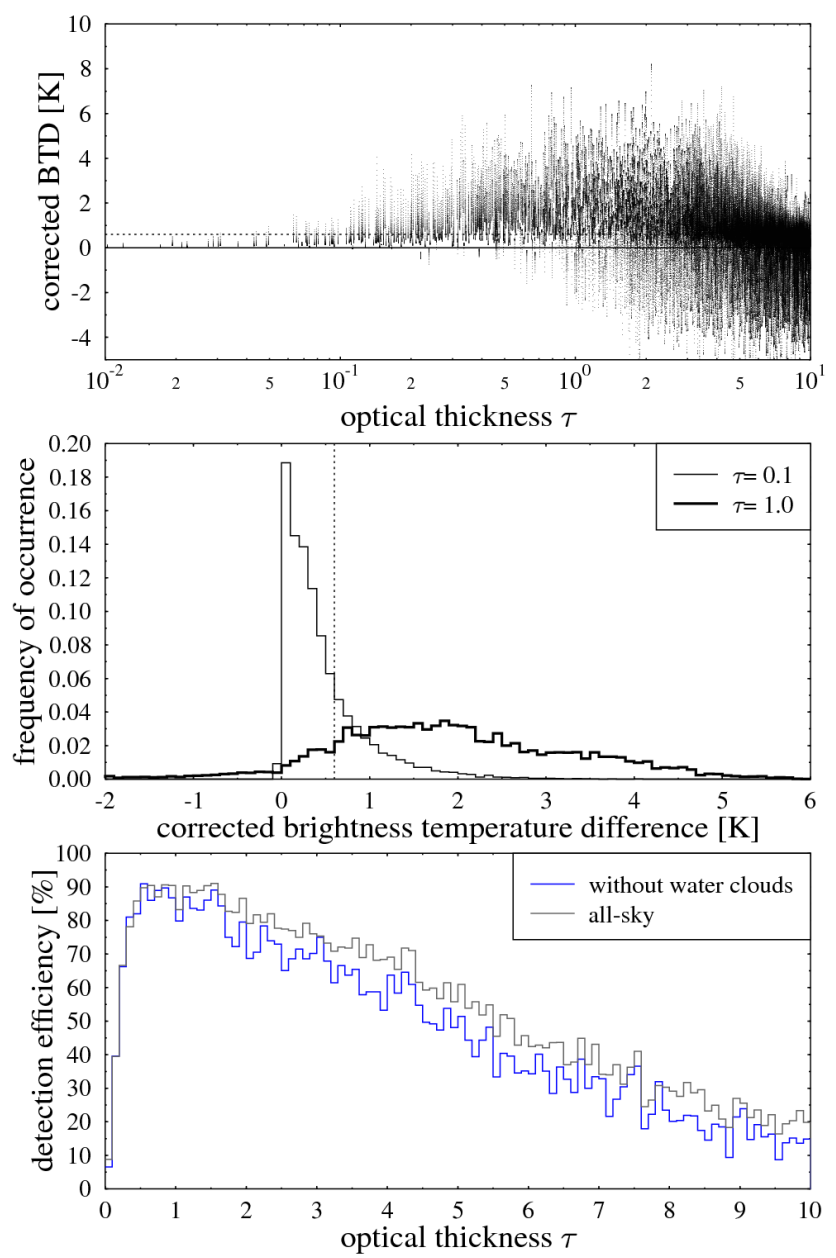

Fig. 4. (Top) Corrected brightness temperature differences of SEVIRI channels IR10.8 and IR12.0 for the 410000 simulated data points of the test data set; the dashed line marks our threshold of $0.6 \mathrm{~K}$; (middle) histogram of corrected brightness temperature differences for two optical thickness ranges, $0-0.2$ (denoted by $\tau=0.1$ ) and 0.9-1.1 (denoted by $\tau=1.0$ ); (bottom) detection efficiency, defined as the fraction of data points correctly classified as cirrus because the corrected BTD was above the threshold; the grey curve includes all data, the blue curve only those without water cloud below.

WV6.2. As shown in Fig. 5, optically thick ice clouds are characterized by a temperature difference larger than $-12 \mathrm{~K}$ which we used as a threshold in MeCiDA (dashed line). This test only identifies optically thicker ice clouds above $6 \mathrm{~km}$ which are missed by the combination of the previous two tests. Jointly, the three tests supposedly identify all kinds of cirrus clouds, see Fig. 3.

A formal description of test 1 together with all other tests is given in Table 1. Also, Fig. 12 shows the result of each individual test for a sample scene, together with the cloud mask obtained from the combination of all tests.

Test 2: split-window 8.7-12 $\mu \mathrm{m}$ and morphology $6.2 \mu \mathrm{m}$

Test 2 also uses the split-window technique, but with a dif-

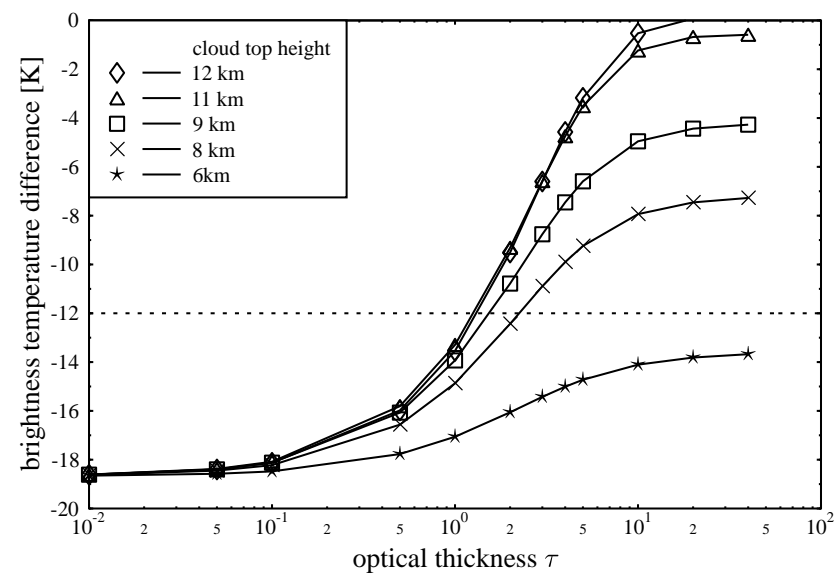

Fig. 5. Simulated brightness temperature difference $T_{6.2}-T_{7.3}$ between SEVIRI water vapor channels WV6.2 and WV7.3 for the mid-latitude summer atmosphere, surface temperature $283 \mathrm{~K}$, a cirrus cloud of geometrical thickness $1 \mathrm{~km}$, effective particle radius $6 \mu \mathrm{m}$, and satellite zenith angle $60^{\circ}$, as a function of optical thickness $\tau$ for five different cloud top altitudes.

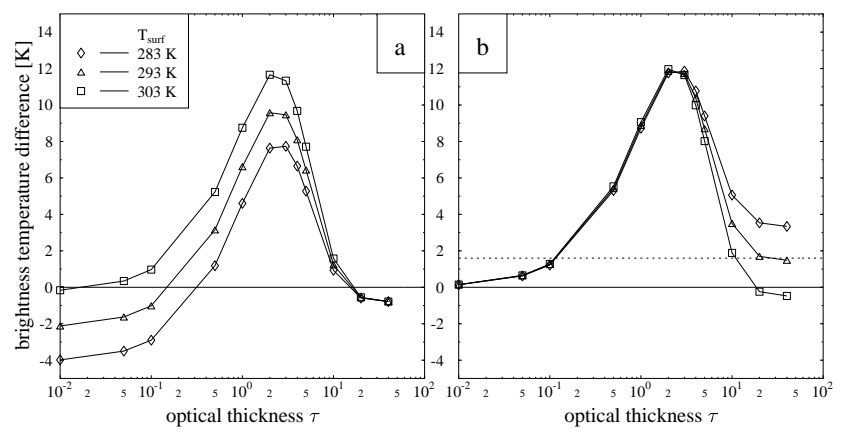

Fig. 6. Simulated brightness temperature difference of SEVIRI channels IR8.7 and IR12.0 for the mid-latitude summer atmosphere, a cirrus cloud between 10 and $11 \mathrm{~km}$, effective particle radius $6 \mu \mathrm{m}$, and satellite zenith angle $60^{\circ}$. (a) Temperature difference as a function of the cloud optical thickness for three different surface temperatures; (b) same, but after subtraction of the respective cloudless temperature difference (= corrected BTD).

ferent combination of channels, IR8.7 and IR12.0. This test is again combined with a morphology test based on the highpass filtered water vapor channel WV6.2 and in conjunction with the absolute temperature difference between the channels IR8.7 and IR10.8. Optically thick ice clouds are added by the same difference of water vapor channels as in test 1 , $T_{6.2}-T_{7.3}$.

Figure 6 a shows the simulated temperature difference. The first part of this test is similar to the semi-transparency test $T_{10.8}-T_{12.0}$, but water vapor absorption in the IR8.7 spectral band is higher than in channels IR10.8 and IR12.0. In consequence, the brightness temperature difference for optically thin cirrus is negative, see Fig. 6a. Ice absorption in channel IR8.7 is also higher than in channel IR12.0. For the test we 
Table 1. Summary of the six cirrus tests and explanation of the filters used.

Test 1

$$
\begin{aligned}
\mathbf{T 1 a}_{\mathbf{n} \times \mathbf{n}}= & {\left[\left(T_{10.8}-T_{12.0}\right)-\left(T_{10.8, \max (n \times n)}-T_{12.0}, \max (n \times n)\right)>0.6 \mathrm{~K}\right] } \\
& \text { AND }\left[T_{7.3, \operatorname{box}(19 \times 19)}-T_{7.3}>0.5 \mathrm{~K}\right]
\end{aligned}
$$

$$
\mathbf{T 1} \mathbf{b}=\left[T_{6.2}-T_{7.3}>-12 \mathrm{~K}\right]
$$

$\mathbf{T 1}=\left[\mathrm{T1a}_{3 \times 3}\right.$ OR $\mathrm{T1}_{\mathbf{9} \times \mathbf{9}}$ OR $\left.\mathrm{T1a}_{19 \times 19}\right]$ OR T1b

Test 2

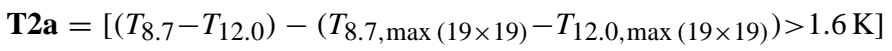

$$
\begin{aligned}
& \text { AND }\left[\left(T_{6.2, \text { box }(19 \times 19)}-T_{6.2}\right)>0.5 \mathrm{~K}\right]
\end{aligned}
$$

$$
\mathbf{T} 2 \mathbf{b}=\left(T_{6.2}-T_{7.3}\right)>-12 \mathrm{~K}
$$$$
\mathbf{T 2} \mathbf{c}=\left(T_{8.7}-T_{10.8}\right)>0 \mathrm{~K}
$$

$\mathbf{T} 2=\left[\begin{array}{lllll}\mathbf{T} 2 \mathbf{a} & \mathrm{OR} & \mathbf{T} 2 \mathbf{b} & \mathrm{OR} & \mathbf{T} 2 \mathbf{c}\end{array}\right]$

Test 3

$$
\begin{aligned}
\text { T3a }= & {\left[\left(T_{9.7}-T_{13.4}\right)-\left(T_{9.7, \max (19 \times 19)}-T_{13.4, \max (19 \times 19)}\right)>3.5 \mathrm{~K}\right] } \\
& \text { AND }\left[\left(T_{7.3, \operatorname{box}(19 \times 19)}-T_{7.3}\right)>0.5 \mathrm{~K}\right]
\end{aligned}
$$

$\mathbf{T 3 b}=\left(T_{6.2}-T_{7.3}\right)>-12 \mathrm{~K}$

$\mathbf{T 3}=\left[\begin{array}{lll}\mathbf{T} 3 \mathbf{a} & \text { OR } & \mathbf{T 3 b}\end{array}\right]$

Test 4

$$
\mathbf{T 4} \mathbf{a}=\left[\left(T_{7.3, \text { box }(15 \times 15)}-T_{7.3}\right)>0.5 \mathrm{~K}\right]
$$

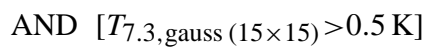

AND $\left[T_{13.4}<253 \mathrm{~K}\right]$

$\mathbf{T 4} \mathbf{b}=T_{13.4}<233 \mathrm{~K}$

$\mathbf{T 4}=\left[\begin{array}{lll}\mathbf{T} 4 \mathbf{a} & \mathrm{OR} & \mathbf{T} 4 \mathbf{b}\end{array}\right]$

$$
\begin{array}{r}
\text { T5a }=\left[\left(T_{6.2}-T 7.3\right)_{\text {box }(15 \times 15)}-\left(T_{6.2}-T_{7.3}\right)>1 \mathrm{~K}\right] \\
\text { AND }\left[\left(T_{6.2}-T_{7.3}\right)_{\text {gauss }}(15 \times 15)>1 \mathrm{~K}\right]
\end{array}
$$

Test 5

$$
\text { AND }\left[T_{13.4}<253 \mathrm{~K}\right]
$$

$\mathbf{T 5} \mathbf{b}=\left[T_{13.4}<233 \mathrm{~K}\right]$

$\mathbf{T 5}=\left[\begin{array}{lll}\mathbf{T 5} \mathbf{a} & \mathrm{OR} & \mathbf{T 5} \mathbf{b}\end{array}\right]$

$$
\text { T6a }=\left[\left(T_{9.7}-T_{13.4}\right)>-7 \mathrm{~K}\right]
$$

Test 6

$$
\text { AND }\left[T_{13.4}<258 \mathrm{~K}\right]
$$

$\mathbf{T 6} \mathbf{b}=T_{13.4}<243 \mathrm{~K}$

$\mathbf{T 6}=\left[\begin{array}{lll}\mathbf{T 6} 6 \mathbf{a} & \mathrm{OR} & \mathbf{T 6 b}\end{array}\right]$

$T_{12.0, \max (n \times n)}$ denotes the maximum brightness temperature in an area of $n \times n$ pixels centered around the pixel under consideration.

Filters $T_{7.3 \text {, box }(n \times n)}$ denotes the average brightness temperature in an area of $n \times n$ pixels centered around the pixel under consideration.

$\left(T_{6.2}-T_{7.3}\right)_{\text {gauss }}(15 \times 15)$ is a Gaussian filter according to Eqs. (5) and (4) 

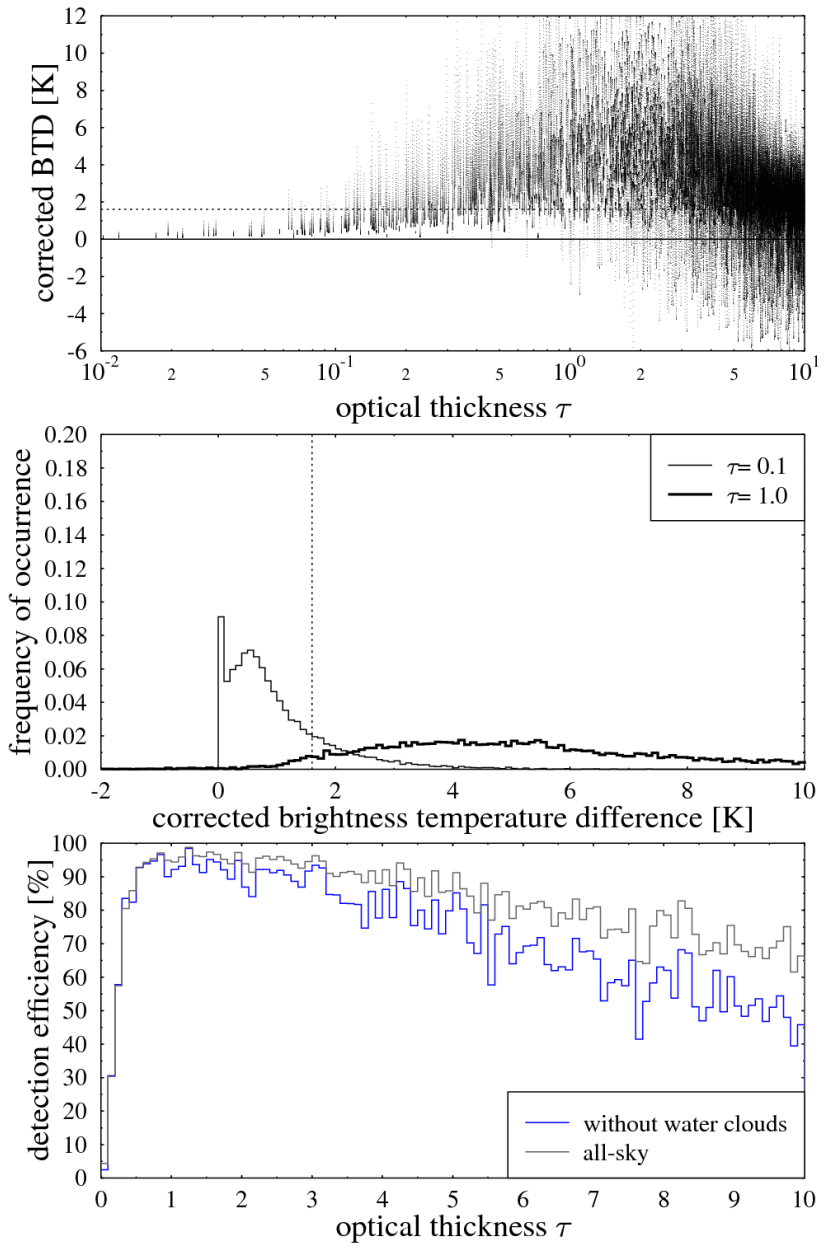

Fig. 7. (Top) Corrected brightness temperature differences of SEVIRI channels IR8.7 and IR12.0 for the 410000 simulated data points of the test data set; the dashed line marks our threshold; (middle) histogram of corrected brightness temperature differences for two optical thickness ranges, $0-0.2$ (denoted by $\tau=0.1$ ) and $0.9-$ 1.1 (denoted by $\tau=1.0$ ); (bottom) detection efficiency, defined as the fraction of data points correctly classified as cirrus because the corrected BTD was above the treshold; the grey curve includes all data, the blue curve only those without water cloud below.

used again the corrected brightness temperature difference (Fig. 6b) with a threshold of $1.6 \mathrm{~K}$.

Figure 7 shows again the application of the split-window test to our test data set. The detection efficiency is close to $100 \%$ for optical thickness between 0.5 and 3 which shows that the combination of IR8.7 and IR12.0 is very efficient for the detection of thin cirrus clouds. In addition, the threshold is considerably larger than in test $1(1.6 \mathrm{~K}$ instead of $0.6 \mathrm{~K})$ which causes the test to be less sensitive to instrumental uncertainties and noise as well as to inhomogeneities in the atmospheric and surface conditions (which affect the determination of the background BTD from nearby cloudless pixels).

This test again might misclassify partially cloudy pixels

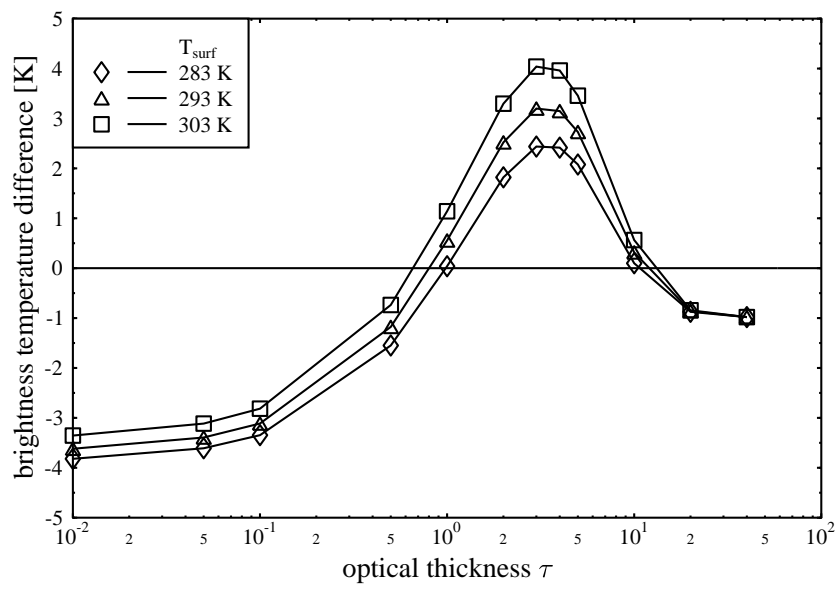

Fig. 8. Simulated brightness temperature difference $T_{8.7}-T_{10.8}$ between SEVIRI infrared channels IR8.7 and IR10.8 for the midlatitude summer atmosphere, a cirrus cloud between 10 and $11 \mathrm{~km}$, effective particle radius $6 \mu \mathrm{m}$, and satellite zenith angle $60^{\circ}$, as a function of optical thickness $\tau$ for three different surface temperatures.

as cirrus, and we actually found misclassification of surface structures in arid areas as cirrus clouds. Therefore the result of the split-window test is combined with the high-pass filtered water vapor channel WV6.2. Water vapor absorption is higher in the spectral band of channel WV6.2 than WV7.3 for which reason this test blocks the surface contribution even more effectively than the corresponding WV7.3 channel in test 1 .

The second part of the test is based on the temperature difference of the channels IR8.7 and IR10.8 (see Fig. 8). Ackerman et al. (1996) suggested this multi-spectral test to detect cirrus from MODIS data. This test is similar to the combination $T_{8.7}-T_{12.0}$ but less sensitive to surface temperature. On the other hand it shows more misclassifications over arid areas. To avoid those a restrictive threshold $T_{8.7}-T_{10.8}>0 \mathrm{~K}$ was introduced. The test is useful for detection of cirrus with an optical thickness between 0.5 and 10 and detects also horizontally widespread cirrus. Finally, we added again the temperature difference $T_{6.2}-T_{7.3}$ to include also optically thick high clouds, see Fig. 5 .

A formal description of test 2 together with all other tests is given in Table 1 .

Test 3: split-window 9.7-13.4 $\mu \mathrm{m}$ and morphology $7.3 \mu \mathrm{m}$

Test 3 is based on the temperature difference between channels IR9.7 and IR13.4. Figure 9a shows the simulated brightness temperature difference between the channels IR9.7 and IR13.4. As in the previous combinations, the brightness temperature difference depends strongly on the surface temperature for thin cirrus clouds and cloudless atmosphere. Low surface temperature results in a high negative difference. Figure $9 \mathrm{~b}$ shows the same, but after subtraction of the respective cloudless temperature difference which 


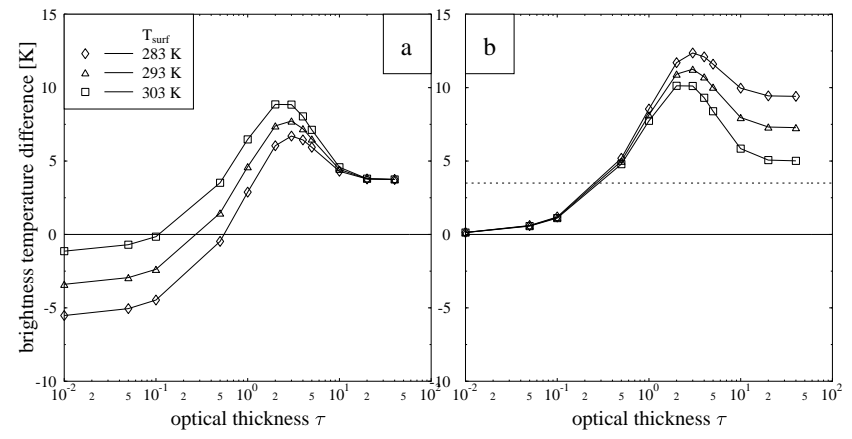

Fig. 9. Simulated brightness temperature difference of SEVIRI channels IR9.7 and IR13.4 for the mid-latitude summer atmosphere, a cirrus cloud between 10 and $11 \mathrm{~km}$, effective particle radius $6 \mu \mathrm{m}$, and satellite zenith angle $60^{\circ}$. (a) Temperature difference as a function of the cloud optical thickness for three different surface temperatures; (b) same, but after subtraction of the respective cloudless temperature difference $(=$ corrected BTD).

is used in test 3. A threshold of $3.5 \mathrm{~K}$ is used in this case (dashed line).

Figure 10 again shows the application of the threshold test to our test data set. The detection efficiency is lower than for the previous tests and the test is mostly sensitive to optically thicker clouds. Application to real data shows that with this channel combination, cirrus clouds have a high contrast not only over the surface but also over low clouds, see Fig. 11. Low clouds appear yellowish in the left (false-color) image. The white or light-blue cirrus clouds in the left image can be easily detected in the temperature difference in the right image applying a constant threshold.

To reduce misclassification of low cloud edges as cirrus clouds, the result is again combined with the high-pass filtered water vapor channel WV7.3, as in test 1. In addition, optically thick ice clouds are detected using the water vapor temperature difference as in both previous tests. A formal description of test 3 together with all other tests is given in Table 1.

\section{Test 4: morphology $7.3 \mu \mathrm{m}$}

Test 4 is a combination of an high-pass filter and a local deviation filter for the water vapor channel WV7.3 and a single channel threshold test using channel IR13.4. In addition to the boxcar filter in the previous three tests, a localised ana$\log$ to the standard deviation is used here. The local deviation filter operation reads as

$g_{i, j}=\sqrt{\left(f_{i, j} \odot K-f_{i, j}\right)^{2} \odot K}$

where $\odot$ is the convolution operator, $f_{i, j}$ is the original image, $K$ is a Gaussian convolution kernel and $g_{i, j}$ is the filtered image. The difference between the original and the
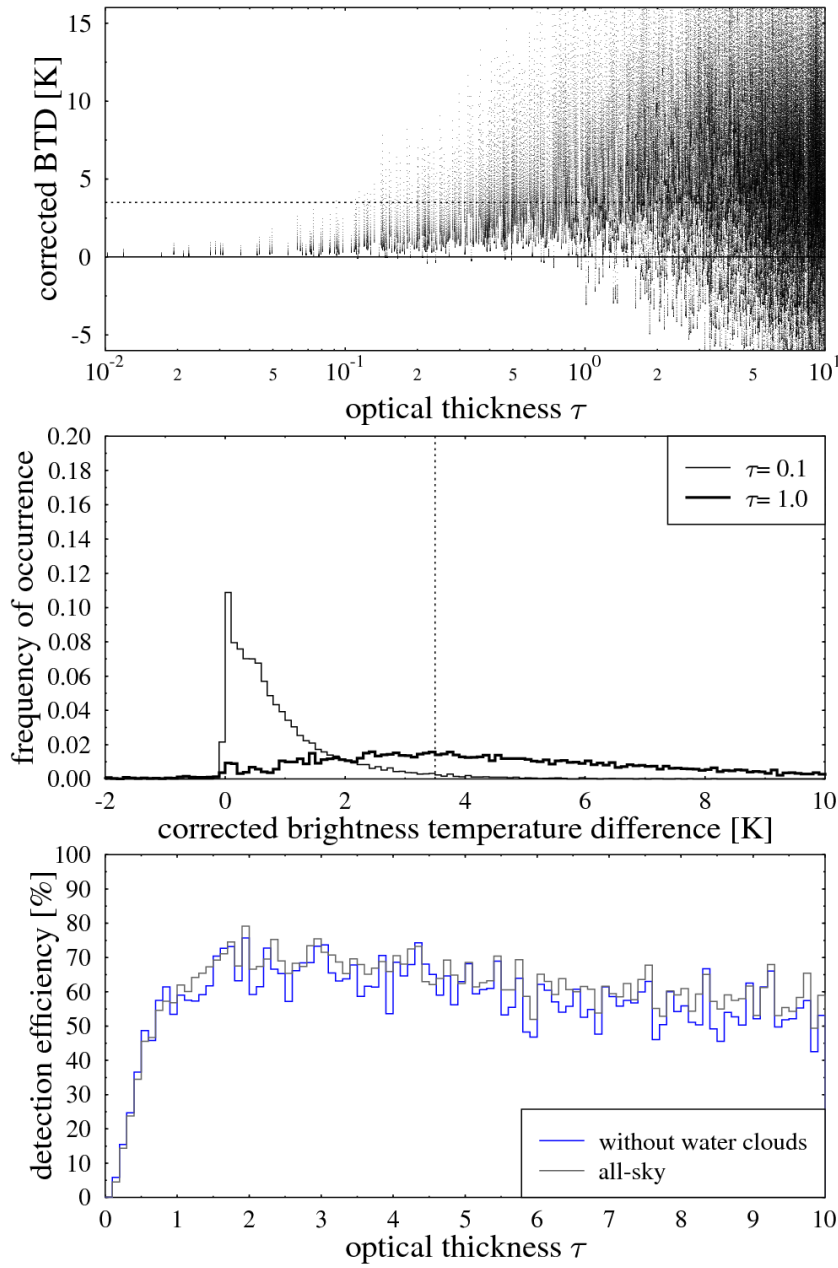

Fig. 10. (Top) Corrected brightness temperature differences of SEVIRI channels IR9.7 and IR13.4 for the 410000 simulated data points of the test data set; the dashed line marks our threshold; (middle) histogram of corrected brightness temperature differences for two optical thickness ranges, $0-0.2$ (denoted by $\tau=0.1$ ) and 0.9 1.1 (denoted by $\tau=1.0$ ); (bottom) detection efficiency, defined as the fraction of data points correctly classified as cirrus because the corrected BTD was above the treshold; the grey curve includes all data, the blue curve only those without water cloud below.

smoothed image is squared and the result is smoothed again. The convolution kernel $K(x, y)$ is

$K(x, y)=\frac{1}{N} \exp \left(-\frac{x^{2}+y^{2}}{2 \cdot\left(\frac{s}{4}\right)^{2}}\right)$

where $N$ is a factor that normalizes the sum of all kernel values to unity, $s$ is the kernel size (an odd number) which we set to 15 for our application, and $x$ and $y$ are in the range from $-(s-1) / 2$ to $+(s-1) / 2$. The advantage of the Gaussian filter compared to the boxcar filter is the rotational symmetry and the reduction of high frequency noise. To further reduce potential mis-classications of variabilities in the water vapor 


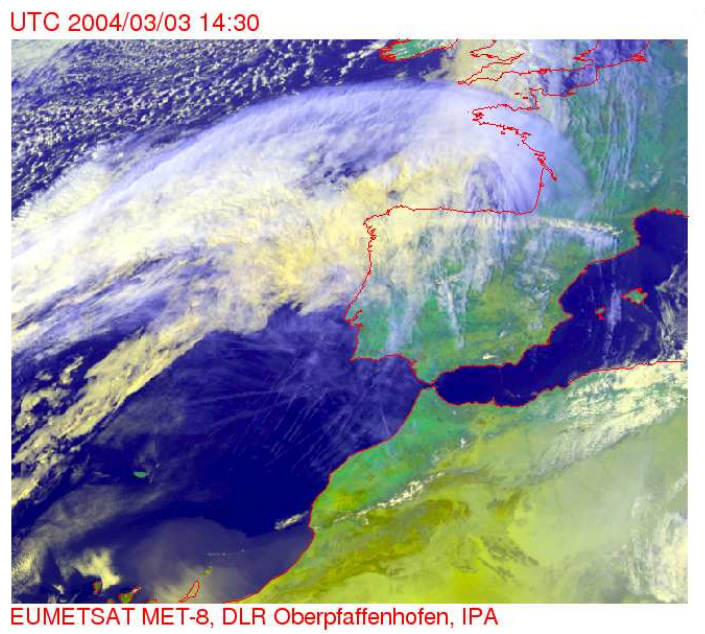

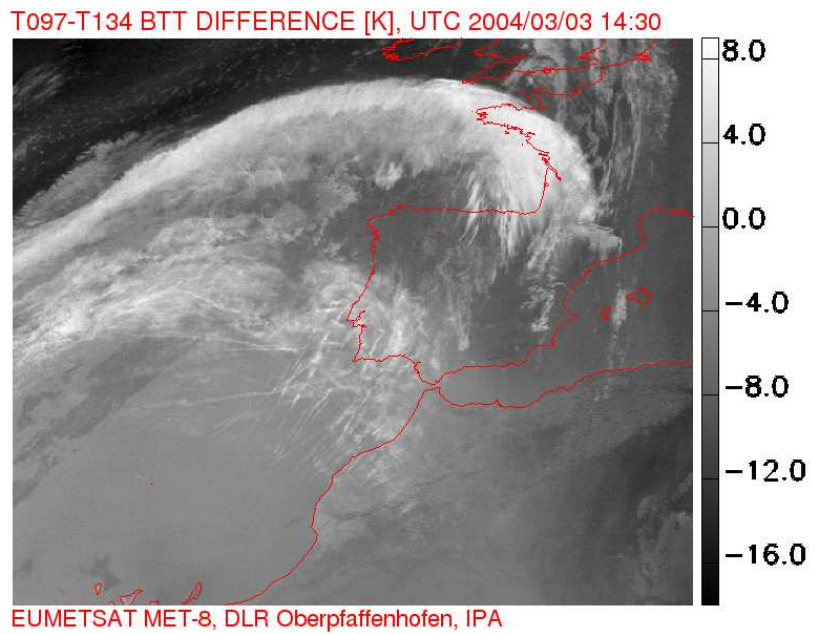

EUMETSAT MET-8, DLR Oberpfaffenhofen, IPA

Fig. 11. (Left) False color composite SEVIRI image over North-West Africa and Western Europe for 3 March 2004, 14:30 UTC; (right) brightness temperature difference $T_{9.7}$ and $T_{13.4}$ between the channels IR9.7 and IR13.4 over North-Western Africa and Western Europe.

field as cirrus cloud, the filtered image is combined with a threshold test for channel IR13.4. To finally include cold and optically thick ice clouds, all pixels with $\mathrm{T}_{13.4}<233 \mathrm{~K}$ are also classified as cirrus.

A formal description of test 4 together with all other tests is given in Table 1

\section{Test 5: morphology in the BTD $6.2 \mu \mathrm{m}-7.3 \mu \mathrm{m}$}

Test 5 is similar to test 4 with the exception that instead of the brightness temperature in channel WV7.3 the temperature difference between the two water vapor channels WV6.2 and WV7.3 is used. While the weighting function of channel WV6.2 is peaked in the upper troposphere and that of channel WV7.3 in the middle troposphere, subtracting the two signals from each other enhances those cloud structures located in the upper part of the atmosphere. In fact, their brightness temperatures are similar in both channels since the amount of water vapor above cloud top is relatively small and the strong ice absorption prevents radiation from lower atmospheric levels from reaching the satellite sensor. In contrast, regions without high clouds show large negative temperature differences $T_{6.2}-T_{7.3}$. The boxcar and the Gaussian filters then extract these structures from the image. Again, to reduce mis-classifications of variabilities in the water vapor field as cirrus cloud, the result of the filtering procedure is combined with a threshold test for channel IR13.4. Cold and optically thick ice clouds are added by means of the simple IR13.4 temperature threshold test $T_{13.4}<233 \mathrm{~K}$. A formal description of test 5 together with all other tests is given in Table 1.

\section{Test 6: BTD 9.7 $\mu \mathrm{m}-13.4 \mu \mathrm{m}$}

Test 6 is a combination of threshold tests based on the brightness temperature difference of channels IR9.7 and IR13.4. This temperature difference depends on satellite zenith angle: With increasing satellite zenith angle, the temperature difference strongly increases. In combination with a threshold test based on channel IR13.4 this test is suitable for cirrus detection in mid-latitudes. Cold and optically thick ice clouds are again detected using the IR13.4 temperature. A formal description of test 6 together with all other tests is given in Table 1.

Finally, all six tests are combined with a logical OR. That is, a pixel is classified as cirrus if at least one of the six tests was positive. The reason for combining various tests was to detect an as large as possible fraction of the cirrus clouds. The six tests are sensitive to different types of cirrus and in combination these tests result in approaching our goal as closely as possible. The thresholds have been chosen to optimize the tests for mid-latitudes and satellite viewing angles up to $75^{\circ}$. Figure 12 shows a sample false color composite of the MSG observations of the Northern Hemisphere and the corresponding cirrus mask. The results of the six individual tests are shown as well to illustrate which test is sensitive to which region of the cirrus clouds. The figure clearly shows that, although there is some redundancy, the six tests provide complementary information. While test 6 for instance correctly identifies the large-scale cirrus it failes to detect the optically thinner parts and in particular the contrails and contrail-cirrus in the lower half of the image. These are very well detected by the split-window tests 1 and 2 . As expected, the morphology test 4 detects mainly highly structured features in the water vapor channel. As for all threshold tests, the performance of the algorithm must be assessed by comparison with independent in-situ or satellite observations. In the next section we present a systematic comparison of our cirrus mask with the respective product of the MODIS instrument on the Terra and Aqua satellites. 


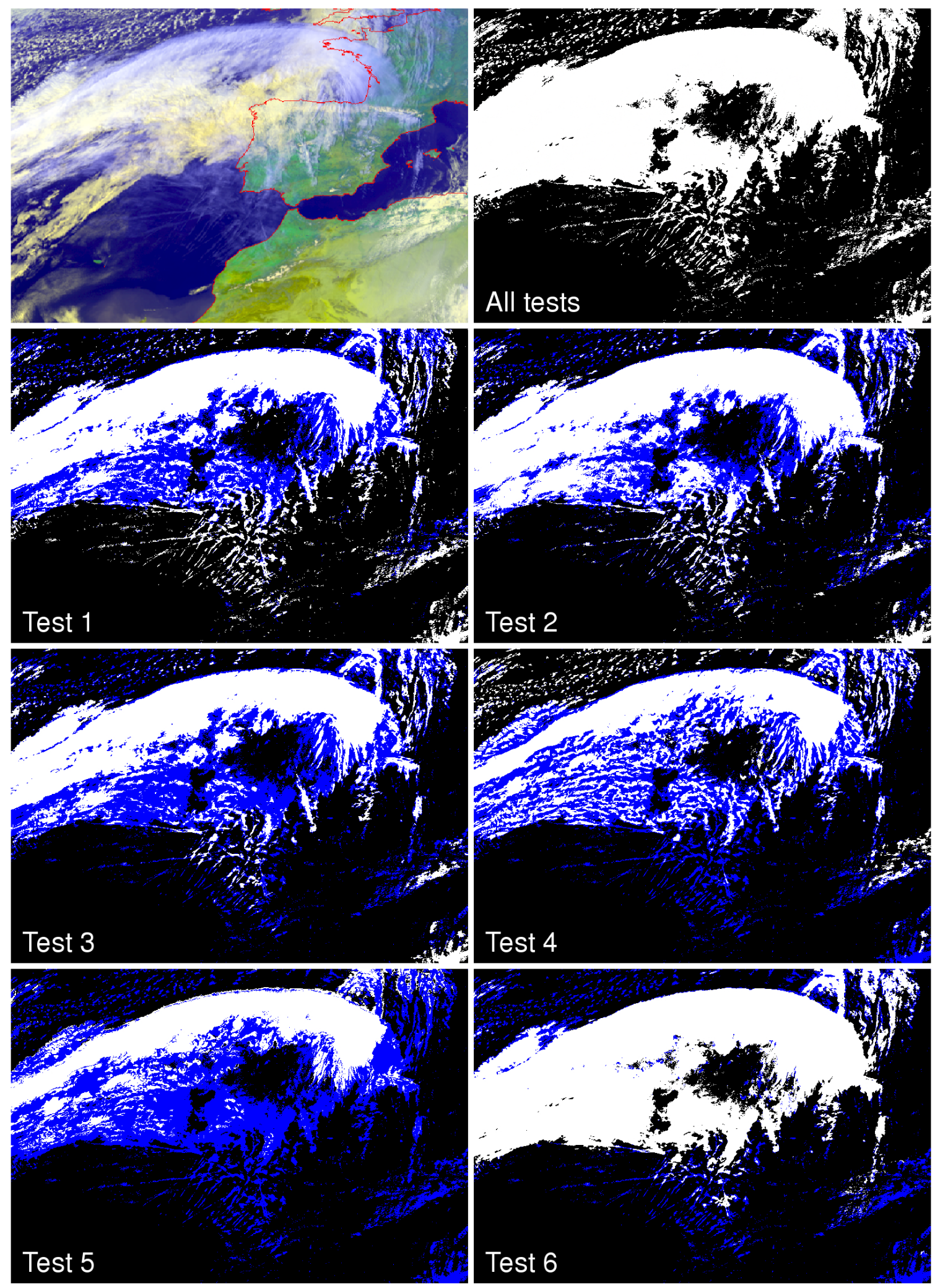

Fig. 12. (Top) False color composite SEVIRI image over North-West Africa and Western Europe for 3 March 2004, 14:30 UTC, and the corresponding MeCiDA cirrus mask (right). The six plots below show the results of the six individual tests described in the text. Shown in white is the result of each test; the blue area is the result of all tests combined which equals the top right image. 


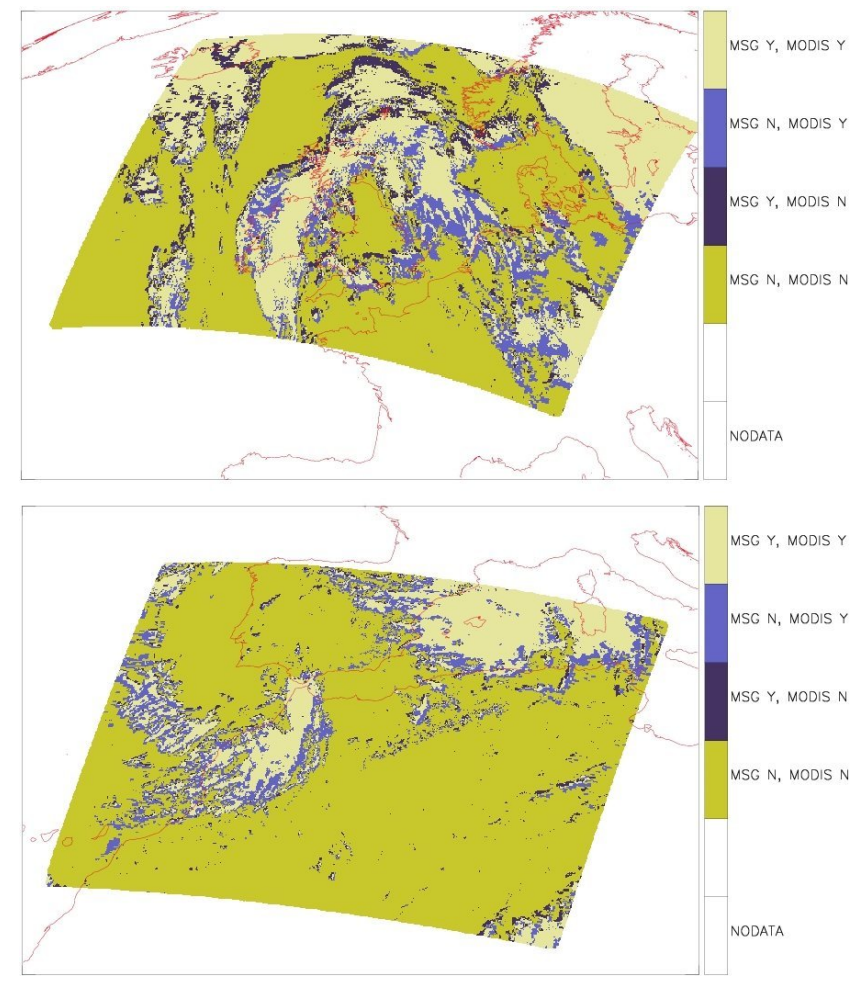

Fig. 13. Comparison between the results of SEVIRI MeCiDA and MODIS-Terra Cirrus Reflectance Flag from the MOD06 L2 product set. (top) 2004/02/12 11:15 UTC; (bottom) 2004/05/31 11:00 UTC. Beige: pixels classified as cirrus by both MeCiDA and MODIS; green: pixels classified as cirrus-free by both MeCiDA and MODIS; light blue: pixels classfied as cirrus by MODIS but as cirrus-free by MeCiDA; dark blue: pixels classified as cirrus by MeCiDA but as cirrus-free by MODIS.

\section{Validation}

The MeCiDA cirrus algorithm has been validated by comparison with MODIS on board the NASA polar obiting Terra and Aqua satellites. MODIS has 36 spectral bands, some of which have been specifically designed to detect thin cirrus clouds. With this spectral information and the high spatial resolution, MODIS is one of the best passive instruments for the detection of cirrus clouds. In addition, the MODIS algorithm also uses the solar channels which we excluded from our algorithm to get consistent results $24 \mathrm{~h}$ per day. MODIS products have been extensively validated by comparison with various independent observations, e.g. the Geoscience Laser Altimeter System GLAS (Mahesh et al., 2004; Hart et al., 2005). For comparison we randomly selected 11 scenes one for each month from February to December, 2004. For the direct comparison we used the "cirrus reflectance flag" from the MOD06L2-collection 004 product (Platnick et al., 2003; King et al., 2003; Ackerman et al., 1998). For this purpose, the MODIS data have been transformed into the satellite projection of SEVIRI. Figure 13 shows two exam-
Table 2. Comparison between MeCiDA results and the MODIS Cirrus Reflectance flag for the year 2004.

\begin{tabular}{ccc}
\hline $\begin{array}{c}\text { Date } \\
\text { dd/mm hh:mm }\end{array}$ & $\begin{array}{c}\text { MeCiDA and MODIS } \\
\text { classified equally }\end{array}$ & $\begin{array}{c}\text { MODIS cirrus } \\
\text { also detected } \\
\text { with MeCiDA }\end{array}$ \\
\hline 02/12 11:15 & $81 \%$ & $71 \%$ \\
03/09 08:45 & $83 \%$ & $60 \%$ \\
04/22 14:00 & $70 \%$ & $39 \%$ \\
05/31 11:00 & $88 \%$ & $59 \%$ \\
06/05 12:45 & $74 \%$ & $50 \%$ \\
$07 / 23$ 09:30 & $99 \%$ & $16 \%$ \\
08/14 14:00 & $91 \%$ & $71 \%$ \\
09/17 12:00 & $80 \%$ & $88 \%$ \\
10/03 10:15 & $80 \%$ & $68 \%$ \\
11/14 11:00 & $78 \%$ & $63 \%$ \\
12/03 09:45 & $62 \%$ & $62 \%$ \\
Average: & $81 \%$ & $60 \%$ \\
\hline
\end{tabular}

ples for the direct comparison over North-Western Africa and Western Europe. The figure shows a good agreement between both classification schemes: green and beige colors are pixels where both algorithms agree on classifying as cirrus or cirrus-free, respectively. Blue colors are mismatches where one of the algorithms detects cirrus while the other doesn't. In particular, close to cloud borders the MODIS detection scheme identifies more cirrus than MeCiDA (light blue). Mismatches at cloud borders might partly be due to wind shift of the cirrus fields and misalignment of both images, as the geolocation of both sensors refers to sea level.

To study possible seasonal variations in the comparison, one MODIS scene was randomly selected for each month between February and December, 2004. The extracted regions cover the whole Northern hemisphere with a focus on Europe and the North-East Atlantic since these areas are our main area of interest. For most scenes good agreement was found between MeCiDA and MODIS, see Table 2. The largest discrepancies appear at high latitudes e.g. close to the coast of Newfoundland, North-Eastern Europe, and Greenland - generally in areas with a large satellite zenith angle for Meteosat Second Generation and a large solar zenith angle in particular for the MODIS scene. Good agreement is found over the North-Sea and Western- and Middle Europe. In summary, MeCiDA and MODIS agreed in $81 \%$ of all pixels in the MODIS field of view. $60 \%$ of the cirrus clouds detected by MODIS were also classified as cirrus by the MeCiDA scheme. A lower detection efficiency is to be expected, due to the lower spatial resolution, the fewer spectral channels, and our restriction to the thermal channels where MODIS uses both solar and thermal channels. Particularly at cloud edges where clouds are optically thinner, MODIS detects more cirrus than SEVIRI. In July, only $16 \%$ of the cirrus 
Table 3. Comparison between monthly means of MeCiDA cirrus coverage and the MODIS Cirrus_Fraction_SWIR for the year 2004 for the region seen by MSG north of $20^{\circ} \mathrm{N}$.

\begin{tabular}{lccc}
\hline Month & MeCiDA cirrus & MODIS cirrus & $\begin{array}{c}\text { correlation } \\
\text { coefficient }\end{array}$ \\
\hline Feb & $43 \%$ & $50 \%$ & 0.80 \\
March & $34 \%$ & $50 \%$ & 0.77 \\
April & $32 \%$ & $45 \%$ & 0.82 \\
May & $28 \%$ & $41 \%$ & 0.84 \\
June & $24 \%$ & $30 \%$ & 0.88 \\
July & $22 \%$ & $25 \%$ & 0.91 \\
Aug & $22 \%$ & $25 \%$ & 0.83 \\
Sep & $22 \%$ & $31 \%$ & 0.74 \\
Okt & $26 \%$ & $38 \%$ & 0.57 \\
Nov & $31 \%$ & $42 \%$ & 0.53 \\
Dec & $40 \%$ & $40 \%$ & 0.40 \\
All: & $29.3 \%$ & $38.1 \%$ & 0.76 \\
\hline
\end{tabular}

detected by MODIS is also detected by MeCiDA. This seemingly large discrepancy is easily explained by the fact that the randomly selected scene was nearly cirrus-free $(99 \%)$ and the small patch of optically thin cirrus was only detected by MODIS.

In addition, monthly means (February 2004-December 2004) of the Cirrus_Fraction_SWIR product from the MYD08 M3 Level 3 collection 004 dataset have been compared to monthly means derived from the MeCiDA results for the area seen by MSG north of $20^{\circ} \mathrm{N}$ see Table 3. The comparison of monthly means shows a better agreement than the comparison of single scenes. This is to be expected because the monthly means provided by the MODIS level 3 products use also the night time cirrus detection which has to rely only on the infrared channels in the same way as the MeCiDA algorithm. For all 11 months MeCida resulted in a cirrus coverage of $29.3 \%$, while MODIS detected $38.1 \%$. In all months the cirrus coverage derived from MODIS data is higher then the coverage derived by MeCiDa. The highest differences occur in the spring and autumn months, while the best correlation is found in summer.

\section{Conclusions}

In this paper, a detailed description of our Meteosat Cirrus Detection Algorithm MeCiDA has been presented. MeCiDA uses only the thermal infrared channels of SEVIRI in order to get a consistent detection scheme at day and night. MeCiDA relies on well-established and new approaches for cirrus identification. Several single and multi-spectral threshold techniques have been used in combination with morphological tests. Of particular use were the water vapor channels which provide separation between lower clouds and cir- rus clouds. Most of the thresholds, in particular those for the split-window tests were determined on the basis of a comprehensive set of radiative transfer calculations. The MeCiDA algorithm is fast enough for near real-time processing. The cirrus detection has been optimized for the Northern part of the Earth visible from MSG (Europe and NorthAtlantic) but it could be adapted to other regions as well. For several tests, "cirrus-free" brightness temperatures are subtracted which are determined from nearby pixels. This requires of course spatial homogeneity of the surface properties. As for all cloud classification schemes, uncertainties are larger over heterogeneous surfaces. A possible future improvement could be the inclusion of satellite derived emissivity maps.

The results have been validated using MODIS derived cirrus coverage data. The comparison shows good results and a high compliance with MODIS derived cirrus masks. MODIS and MeCiDA classified $81 \%$ of the pixels identically, either as cirrus or cirrus-free. MeCiDA detected about $60 \%$ of the MODIS cirrus clouds. The lower detection efficiency (or higher threshold optical thickness) is to be expected considering the better spatial and spectral resolution of MODIS and its use of the solar channels. The advantage of the MeCiDA product is its availability every $15 \mathrm{~min}$, consistently for day and night. The high temporal resolution $(15 \mathrm{~min}$ ) facilitates the investigation of diurnal variations and life cycle aspects of cirrus clouds and will help to improve the understanding of their impact on the radiation balance and climate of the Earth.

Acknowledgements. The authors thank all members of the ESADUE (Data User Element) project CONTRAILS into which this work was embedded. In particular, we would like to thank S. Pinnock (ESA), the technical officer of the CONTRAILS project. We acknowledge the easy access to MODIS data provided by NASA and the MODIS team. We also thank C. Forster for carefully reading the manuscript and helpful discussion which increased the quality of the manuscript. We are grateful to two reviewers who helped to considerably improve the clarity of the presentation with their detailed comments. Finally, we thank K. Graf for his help in preparing the figures.

Edited by: R. MacKenzie

\section{References}

Ackerman, S., Strabala, K., Frey, R., Moeller, C., and Menzel, W.: Cloud Mask for the MODIS Airborne Simulator (MAS): Preparation for MODIS, in: Eighths AMS conference on Satellite Meteorology and Oceanography, 28 January-2 February , 1996, Atlanta, Georgia, pp. 317-320, Am. Meteorol. Soc., 1996.

Ackerman, S., Strabala, K., Menzel, W., Frey, R., Moeller, C., and Gumley, L.: Discriminating clear sky from clouds with MODIS, J. Geophys. Res., 103, 32 141-32 157, 1998.

Anderson, G. and Hall, L.: Solar Irradiance between 2000 and 3100 Angstroms With Spectral Band Pass of 1.0 Angstroms, J. Geophys. Res., 94, 6435-6441, 1989. 
Chevallier, F., Cheruy, F., Scott, N., and Chedin, A.: A neural network approach for a fast and accurate computation of a longwave radiative budget, J. Appl. Meteorol., 37, 1385-1397, 1998.

Derrien, M. and Le Gleau, H.: MSG/SEVIRI cloud mask and type from SAFNWC, Int. J. Remote Sens., 26, 4707-4732, 2005.

Feijt, A., de Valk, P., and van der Veen, S.: Cloud detection using Meteosat imagery and numerical weather prediction model data, J. Appl. Meteorol., 39, 1017-1030, 2000.

Fu, Q., Lesins, G., Higgins, J., Charlock, T., Chylek, P., and Michalsky, J.: Broadband water vapour absorption of solar radiation tested using ARM data, Geophys. Res. Lett., 25, 1169-1172, 1998.

Hansen, J., Sato, M., and Ruedy, R.: Radiative forcing and climate response, J. Geophys. Res., 102, 6831-6864, 1997.

Hart, W., Spinhirne, J., Palm, S., and Hlavka, D.: Height distribution between cloud and aerosol layers from the GLAS spaceborne lidar in the Indian Ocean region, Geophys. Res. Lett., 32, doi:10.1029/2005GL023 671, 2005.

Inoue, T.: On the temperature and effective emissivity determination of semitransparent cirrus clouds by bispectral measurements in the $10 \mu \mathrm{m}$ window region, J. Meteor. Soc. Japan, 63, 88-98, 1985.

IPCC: Climate Change 2007: The Scientific Basis, Tech. rep., Intergovernmental Panel on Climate Change (IPCC), IPCC Secretariat, c/o World Meteorological Organization, Geneva, Switzerland, 2007.

Kidder, S. and Vonder Haar, T.: Satellite Meteorology, Academic Press, 1995.

King, M., Menel, W., Kaufman, Y., Tanre, D., Gao, B.-C., Platnick, S., Ackerman, S., Remer, L., Pincus, R., and Hubanks, P.: Cloud and aerosol properties, precipitable water, and profiles of temperature and water vapor from MODIS, IEEE Transactions on Geoscience and Remote Sensing, 41, 442-458, 2003.

Kriebel, K.-T., Gesell, G., Kästner, M., and Mannstein, H.: The cloud analysis tool APOLLO: improvements and validation, Int. J. Remote Sens., 24, 2389-2408, 2003.

Lynch, D., Sassen, K., Starr, D., and Stephens, G.: Cirrus, Oxford University Press, 3-10, 2002.

Mahesh, A., Gray, M., Palm, S., Hart, W., and Spinhirne, J.: Passive and active detection of clouds: Comparisons between MODIS and GLAS observations, Geophys. Res. Lett., 31, doi:10.1029/2003GL018 859, 2004.

Mayer, B. and Kylling, A.: Technical Note: The libRadtran software package for radiative transfer calculations: Description and examples of use, Atmos. Chem. Phys., 5, 1855-1877, 2005, http://www.atmos-chem-phys.net/5/1855/2005/.

Mayer, B., Seckmeyer, G., and Kylling, A.: Systematic long-term comparison of spectral UV measurements and UVSPEC modeling results, J. Geophys. Res., 102, 8755-8767, 1997.
Meerkötter, R., Schumann, U., Doelling, D., Minnis, P., Nakajima, T., and Tsushima, Y.: Radiative forcing by contrails, Ann. Geophys., 17, 1080-1094, 1999, http://www.ann-geophys.net/17/1080/1999/.

Minnis, P. and Smith Jr., W.: Cloud and radiative fields derived from GOES-8 during SUCCESS and the ARM-UAV spring 1996 flight series, Geophys. Res. Lett., 25, 1113-1116, 1998.

Pierluissi, J. and Peng, G.-S.: New molecular transmission band models for LOWTRAN, Opt. Engin., 24, 541-547, 1985.

Platnick, S., King, M., Ackerman, S., Menzel, W., Baum, B., Riedi, J., and Frey, R.: The MODIS cloud products: Algorithms and examples from TERRA, IEEE Transactions on Geoscience and Remote Sensing, 41, 459-473, 2003.

Ricchiazzi, P. and Gautier, C.: Investigation of the effect of surface heterogeneity and topography on the radiation environment of Palmer Station, Antarctica, with a hybrid 3-D radiative transfer model, J. Geophys. Res., 103, 6161-6178, 1998.

Rossow, W. and Schiffer, R.: Advances in understanding clouds from ISCCP, B. Am. Meteorol. Soc., 80, 2261-2287, 1999.

Saunders, R. and Kriebel, K.: An improved method for detecting clear sky and cloudy radiances from AVHRR data, Int. J. Remote Sens., 9, 123-150, 1988.

Schmetz, J., Pili, P., Tjemkes, S., Just, D., Kerkmann, J., Rota, S., and Ratier, A.: An introduction to Meteosat Second Generation (MSG), B. Am. Meteorol. Soc., pp. 977-992, 2002.

Stamnes, K., Tsay, S., Wiscombe, W., and Jayaweera, K.: A numerically stable algorithm for discrete-ordinate-method radiative transfer in multiple scattering and emitting layered media, Appl. Opt., 27, 2502-2509, 1988.

Van Weele, M., Martin, T., Blumthaler, M., Brogniez, C., den Outer, P., Engelsen, O., Lenoble, J., Pfister, G., Ruggaber, A., Walravens, B., Weihs, P., Dieter, H., Gardiner, B., Gillotay, D., Kylling, A., Mayer, B., Seckmeyer, G., and Wauben, W.: From model intercomparisons towards benchmark UV spectra for six real atmospheric cases, J. Geophys. Res., 105, 4915-4925, 2000.

World Meteorological Organization: International Cloud Atlas. Volume I - Manual on the observation of clouds and other meteors, WMO, 1975.

World Meteorological Organization: International Cloud Atlas. Volume II - Plates, WMO, 1987.

Wylie, D. and Woolf, H.: The Diurnal Cycle of Upper-Tropospheric Clouds Measured by GOES-VAS and the ISCCP, Mon. Weather Rev., 130, 171-179, 2002.

Wylie, D., Jackson, D., Menzel, W., and Bates, J.: Trends in global cloud cover in two decades of HIRS observations, J. Clim., 18, 3021-3031, 2005 